\title{
THE MAGNETIC PROPERTIES OF SUPERCONDUCTING ALLOYS I
}

\author{
KAZUMI MAKI \\ Research Institute for Mathematical Sciences, Kyoto University, Kyoto
}

(Received 27 April 1964)

\begin{abstract}
The general Ginzburg - Landau equation valid for all temperatures is derived from the microscopic theory for superconducting alloys where the electronic mean free path 1 is much shorter than the coherence length $\xi_{0}$.

Using this equation the magnetic properties of Abrikosov's mixed state as well as the temperature dependence of the upper critical field $\mathrm{H}_{\mathrm{c}_{2}}$ are discussed. It is shown that the Abrikosov structure in the vicinity of the transition point is completely characterized by two parameters $\kappa_{1}(\mathrm{~T})$ and $\kappa_{2}(\mathrm{~T})$, both of which coincide at the critical temperature with $\kappa$, the Ginzburg-Landau parameter. A peculiar deviation in the thermodynamical behavior at lower temperatures from Abrikosov's theory is found for superconductors with $\kappa \simeq 1 / \sqrt{2}$.
\end{abstract}

\section{Introduction}

AS is well known, the behavior of superconducting alloys in a magnetic field is significantly different from that of pure superconductors. Based on the semi-phenomenological Ginzburg - Landau equation Abrikosov [1] has investigated the magnetic properties of type II superconductors and shown the existence of mixed states, where the superconductivity is partially destroyed and the magnetic field penetrates into the bulk as an array of quantized flux lines. It is interesting to see if his theory holds at lower temperatures where the GinzburgLandau equation is no longer valid [2].

Using a technique similar to that developed by Nambu and Tuan [3] in the study of the field dependence of the gap parameter, Tewordt [4] obtained an interesting extension of the Ginzburg - Landau equation, which seems, however, applicable only to the weak field case. Werthamer [5] arrived at the same result in a somewhat different way.

In the present paper we derive a general Ginzburg - Landau equation valid at all temperatures on the assumption that the electronic mean free path 1 is very short $\left(1 / \xi_{0} \ll 1\right.$ where $\xi_{0}$ is the coherence length), expanding the Gor'kov equation [6] in powers of the ordering parameter $\Delta(x)$.

In the case $1 / \xi_{0} \ll 1$ the magnetic field is introduced into the theory by a simple replacement $i \nabla$ $\longrightarrow i \nabla \pm 2 \mathrm{e} A$, where $A$ is the vector potential. The set of equations thus obtained enables us to discuss the magnetic properties of superconductors in the transition region independently of temperature.

Previously Gor'kov [7] and Shapoval [8] calculated the upper critical field $H_{\mathrm{c} 2}$ from the microscopic theory. The latter, especially, considered the case of alloys where the electronic mean free path is short $\left(1 / \xi_{0} \ll 1\right)$. A detailed experimental study of the temperature dependence of $H_{c 2}$ has been recently carried out by Kinsel, Lynton and Serin [10] on indium based alloys. They pointed out that their results are in good agreement with Gor'kov's theory but disagree with Shapoval's. The present investigation was initiated to solve this apparent discrepancy. The upper critical field differs remarkably from Shapoval's at lower temperatures. Since the beginning of this work, Werthamer [9] pointed out that there is a numerical error in Shapoval's calculation which, when corrected, brings it into agreement with Gor'kov's. Our result is not widely different from Gor'kov's,

The Abrikosov structure [1] in the vicinity of the transition point is determined by the use of a variational method. It is shown that the structure is characterized by two parameters $\kappa_{1}(T)$ and $\kappa_{2}(T)$ where 
$\kappa_{1}(T)=H_{c 2} / \sqrt{2 H_{c}}$ and $\kappa_{2}(T)$ appears in the expression for the magnetization $M$ as $-4 \pi M /\left(H_{c 2}-H_{0}\right)$ $=1 / 1.18\left(2 \kappa_{2}{ }^{2}-1\right)$ where $H_{0}$ is the external field. The temperature dependence of both parameters is calculated and we find the following relation: $\kappa_{1}(T) \geqq \kappa \geqq \kappa_{2}(T)$, where the equality holds only at $T=T_{c 0}$.

An interesting deviation from Abrikosov's theory is predicted for superconductors with $\kappa_{1}$ and $\kappa_{2}$ satisfying $\kappa_{1}>1 / \sqrt{2}>\kappa_{2}$.

\section{The General Ginzburg - Landau Equation}

In this section we are concerned with the derivation of equations for the ordering parameter $\Delta$ and the current $\bar{j}$ valid at arbitrary temperatures. We assume here only the smallness of the ordering parameter (i.e., $\left.\Delta / \pi T_{c 0} \ll 1\right)$, since we are interested in the magnetic properties of superconducting alloys close to the transition point. The method used in the following is a simple extension of one employed by Gor'kov [2] in the microscopic derivation of the Ginzburg-Landau theory.

To begin with we consider an impurity-free superconductor in the absence of magnetic fields. According to Gor'kov [2] we obtain the following equation for the ordering parameter $\Delta$ when $\Delta$ is small:

$$
\begin{aligned}
& \begin{aligned}
\Delta^{+}(r)= & g T \sum_{n} \int G_{\omega}\left(r^{\prime}, r\right) G_{-\omega}\left(r, r^{\prime}\right) \Delta^{+}\left(r^{\prime}\right) d^{3} r^{\prime} \\
& -g T \sum_{n} \iiint G_{\omega}(s, r) G_{-\omega}(s, I) G_{\omega}(m, I) G_{-\omega}(m, r) \\
& \times \Delta^{+}(s) \Delta(I) \Delta^{+}(m) d^{3} s d^{3} l d^{3} m,
\end{aligned} \\
& \left.\omega=\pi T(2 n+1) \quad \text { and } \quad \Delta(r)=|g|<T \psi_{\uparrow}^{+}(r, t) \psi_{\downarrow}^{+}(r, t)\right\rangle,
\end{aligned}
$$

where $G_{\omega}\left(r, r^{\prime}\right)$ is the Green's function of the electrons in the normal metal, whose Fourier transform is $(i \omega-\xi)^{-1}$ with $\xi=p^{2} / 2 m^{-\mu}$. The effect of impurity scattering is most easily included in the theory by the use of the standard techniques in the theory of metals. In the presence of impurity atoms one has, firstly, to use instead of the Green's function in a pure metal the modified Green's function, which is obtained from the

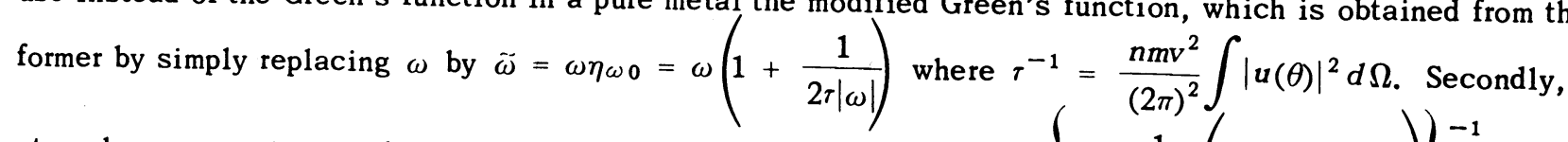
at each proper vertex one has to multiply by a factor $\eta_{\omega_{q}}$ where $\eta_{\omega_{q}}=\left\{1-\frac{1}{2 \tau \widetilde{\omega}}\left(1-\frac{\pi \tau_{t r}}{3} v^{2} q^{2}\right)\right\}^{-1}, \vec{q}$ is the external momentum and $v$ the fermi velocity; one has to replace $\Delta_{q}$ (or $\Delta_{q}^{+}$) by $\eta_{\omega_{q}} \Delta_{q}$ (or $\eta_{\omega_{q}} \Delta_{q}^{+}$) where $\Delta_{q}$ is the Fourier transform of $\Delta(x) . \tau_{t r}$ is the relaxation time for transport. Thus we arrive at the following
formal equation

$$
\begin{aligned}
\Delta^{+}(r)= & \frac{m p_{0}|g|}{2 \pi^{2}}\left[2 \pi T \sum_{n}^{\omega_{D}} \frac{1}{2 \omega+\frac{\tau_{t r}}{3} v^{2} q^{2}} \Delta^{+}(r)\right. \\
& \left.-\left.2 \pi T \sum_{n} \omega\left(\prod_{i=1}^{4} \frac{1}{2 \omega+\frac{\tau_{t r}}{3} v^{2} q_{i}^{2}}\right) \Delta^{+}(1) \Delta(2) \Delta^{+}(3)\right|_{1=2=3=r}\right],
\end{aligned}
$$
where $\sum_{i=1}^{4} \vec{q}_{i}=0$ and $\vec{q}_{i}$ operates only on $\Delta(i)$. Making use of the identity $1=\frac{m p_{0}|g|}{2 \pi^{2}} \ln \left(\frac{\gamma \omega_{D}}{\pi T_{\mathrm{c} 0}}\right)$ where
$T_{\mathrm{c} 0}$ is the critical temperature we further simplify equation (2) 


$$
\begin{aligned}
\left\{\ln \frac{T}{T_{c 0}}+f_{0}\left(\frac{\tau_{t r} v^{2} q^{2}}{12 \pi T}\right)\right\} \Delta^{+}(r) & \\
& +\left.\frac{1}{8(\pi T)^{2}} \sum_{n}\left(n+\frac{1}{2}\right)\left(\prod_{i=1}^{4} \frac{1}{n+\frac{1}{2}+\frac{\tau_{t r} v^{2} q_{i}^{2}}{12 \pi T}}\right) \Delta^{+}(1) \Delta(2) \Delta^{+}(3)\right|_{1=2=3=r}=0,
\end{aligned}
$$

where $f_{0}(z)=\psi\left(\frac{1}{2}+z-\psi\left(\frac{1}{2}\right)\right.$ and $\psi(z)=\Gamma^{\prime}(z) / \Gamma(z)$ is the di-gamma function. The first term is just the equation used by Werthamer [9] in his study of the proximity effect. A similar procedure leads us to the following expression for the current density:

$$
j(r)=-\left.\frac{e \tau_{t r} N}{4 m \pi T} \sum_{n}\left(\prod_{i=1}^{2} \frac{1}{n+\frac{1}{2}+\frac{\tau_{t r} v^{2} q^{2}}{12 \pi T}}\right)\left(\vec{q}_{1}-\vec{q}_{2}\right) \Delta(1) \Delta^{+}(2)\right|_{1=2=r}
$$

Now we will consider the necessary modifications of both equations (3) and (4) in the presence of a magnetic field. As is well known $\Delta^{+}$changes into $e^{2 i e \lambda} \Delta^{+}$in the usual guage transformation $\psi \longrightarrow e^{-i e \lambda} \psi$ and the gauge invariance of both equations (3) and (4) is most easily guaranteed if one replaces $q$ in equations (3) and (4) by $\vec{q}-2 e \vec{A}$ or $\vec{q}+2 e \vec{A}$ depending on whether it operates on $\Delta^{+}$or $\Delta$. The requirement of gauge invariance is not sufficient to determine uniquely the field-dependent terms. We can add any terms containing $\vec{H}=\nabla \times \vec{A}$ without conflicting with this requirement. In the appendix we show explicitly that any such terms are of the order of $1 / \xi_{0}$, and they are neglected in the present discussion. The basic equations are given as

$$
\begin{aligned}
& \left\{\ln \frac{T}{T_{c 0}}+f_{0}\left[\frac{\tau_{t r} v^{2}}{12 \pi T}(\vec{q}-2 e \vec{A})^{2}\right]\right\} \Delta^{+}(r) \\
& \quad+\left.\frac{1}{8(\pi T)^{2}} \sum_{n}\left(n+\frac{1}{2}\right)\left(\prod_{i=1}^{4} X_{n i}^{-1}\right) \Delta^{+}(1) \Delta(2) \Delta_{1=2=3=r}^{+}(3)\right|_{1=2}=0,
\end{aligned}
$$

and

$$
j(r)=-\left.\frac{e \tau_{t r} N}{4 m \pi T} \sum_{n}\left(\prod_{i=1}^{2} X_{n i}{ }^{-1}\right)\left(\vec{q}_{1}-\vec{q}_{2}+4 e \vec{A}\right) \Delta^{+}(1) \Delta(2)\right|_{1=2=r},
$$

where

$$
X_{n i}=n+\frac{1}{2}+\frac{\tau_{t r} v^{2}}{12 \pi T}\left(\vec{q}_{i}-(-1)^{i} 2 \mathrm{e} \vec{A}\right)^{2}
$$

which correspond to the equations of the Ginzburg - Landau theory. In the vicinity of the critical temperature where $\frac{\tau_{t r} v^{2}}{12 \pi T}(\vec{q} \pm 2 e \vec{A})^{2}$ is small the above equations reduce to those obtained by Gor'kov [2].

\section{The Upper Critical Field}

Gor'kov [7] has shown that there is another critical field, besides the usual thermodynamical critical field $H_{c}$, corresponding to the limiting field below which the normal phase becomes unstable against the formation of a local superconducting correlation and calculated this field (the upper critical field $H_{c_{2} \text { ) }}$ 
using the linearized equation for $\Delta$. Shapoval [8] extended his treatment to the case of superconducting alloys where the electronic mean free path is very short $\left(1 / \xi_{0} \ll 1\right)$. They included the effect of a magnetic field in the theory simply by multiplying the integral kernel by a phase factor. As the treatment of magnetic fields in the present formulation is considerably different from theirs, it is of interest to derive anew the upper critical field. For this purpose it is sufficient to consider the linear part of equation (5). In the following we take the direction of the magnetic field as the $z$ axis. In a uniform magnetic field we obtain

$$
\left\{\ln \frac{T}{T_{\mathrm{c} 0}}+f_{0}\left(-\frac{\tau_{t r} v^{2}}{12 \pi T}\left(\frac{\partial^{2}}{\partial x^{2}}-4 e^{2} H^{2} x^{2}\right)\right)\right\} \Delta(x)=0,
$$

where we put $\vec{A}=(0, H x, 0)$ and assume $\Delta$ depends only on $x$. The upper critical field is determined as the maximum eigenvalue $H$ of the above equation. It is easy to solve this equation and we find

$$
\ln \frac{T}{T_{c 0}}+f_{0}\left(\frac{\tau_{t r} v^{2}}{6 \pi T} e H\right)=0
$$

and

$$
\Delta(x)=e^{-e H x^{2}}
$$

Using the asymptotic expression for $\psi(z)$ we obtain

$$
\begin{aligned}
H_{\mathrm{c} 2} & =\frac{3}{2} \frac{\Delta_{00}}{\tau_{t r} v^{2} \mathrm{e}}\left[1-\frac{2}{3}\left(\frac{\pi T}{\Delta_{00}}\right)^{2}\right], \quad \text { for } \quad T \ll T_{\mathrm{c} 0}, \\
& =\frac{12 T_{\mathrm{c} 0}}{\pi \tau_{t r} v^{2} \mathrm{e}}\left(1-\frac{T}{T_{\mathrm{c} 0}}\right)\left[1-\left(\frac{1}{2}-\frac{28}{\pi^{4}} \zeta(3)\right) \theta\right], \quad \text { for } \quad T_{\mathrm{c} 0}-T \ll T_{\mathrm{c} 0},
\end{aligned}
$$

where $\theta=1-T / T_{\mathrm{c} 0}$.

Or defining a new parameter $\kappa_{1}$ as $\kappa_{1}=H_{\mathrm{c} 2}(T) / \sqrt{2} H_{\mathrm{c}}(T)$,

$$
\begin{aligned}
\kappa_{1}(T) & =\frac{\pi^{2}}{2 \sqrt{2}}(7 \xi(3))^{-1 / 2} \kappa\left[1-\frac{1}{3}\left(\frac{\pi T}{\Delta_{00}}\right)^{2}\right], \\
& =1.20 \kappa\left(1-1.06 \frac{T^{2}}{T_{\mathrm{c} 0}^{2}}\right), \quad \text { for } \quad T \ll T_{c 0}, \\
& =\kappa(1+0.391 \theta), \quad \text { for } \quad T_{\mathrm{c} 0}-T \ll T_{\mathrm{c} 0},
\end{aligned}
$$

where $\kappa=\frac{3 m}{2 \pi^{2} \mathrm{e} \tau_{t r}}\left(\frac{2 \pi m}{p_{0}{ }^{5}} 7 \xi(3)\right)^{1 / 2}$ is the Ginzburg - Landau parameter first calculated by Gor'kov [2].

The above results are depicted in Fig. 1. together with the results of Gor'kov [7] and Shapoval [8]. One sees from the figure that our theory predicts a somewhat smaller value of $H_{c 2}$ than Gor'kov's and the devia-
tion from Shapoval's at lower temperatura latter discrepancy has been stated by Wer is remarkable. As remarked in the introduction, the origin of this Shapoval.

\section{Abrikosov's Mixed State}

When the magnetic field is slightly smaller than $H_{c_{2}}$ and the ordering parameter $\Delta$ is small, it is expected that one can discuss the behavior of the superconducting phase by the use of equations (5) and (6).
As is shown by Abrikosov [1] the the state) in a strong magnetic field. This state appears at a low is brought into a new state (Abrikosov's mixed 


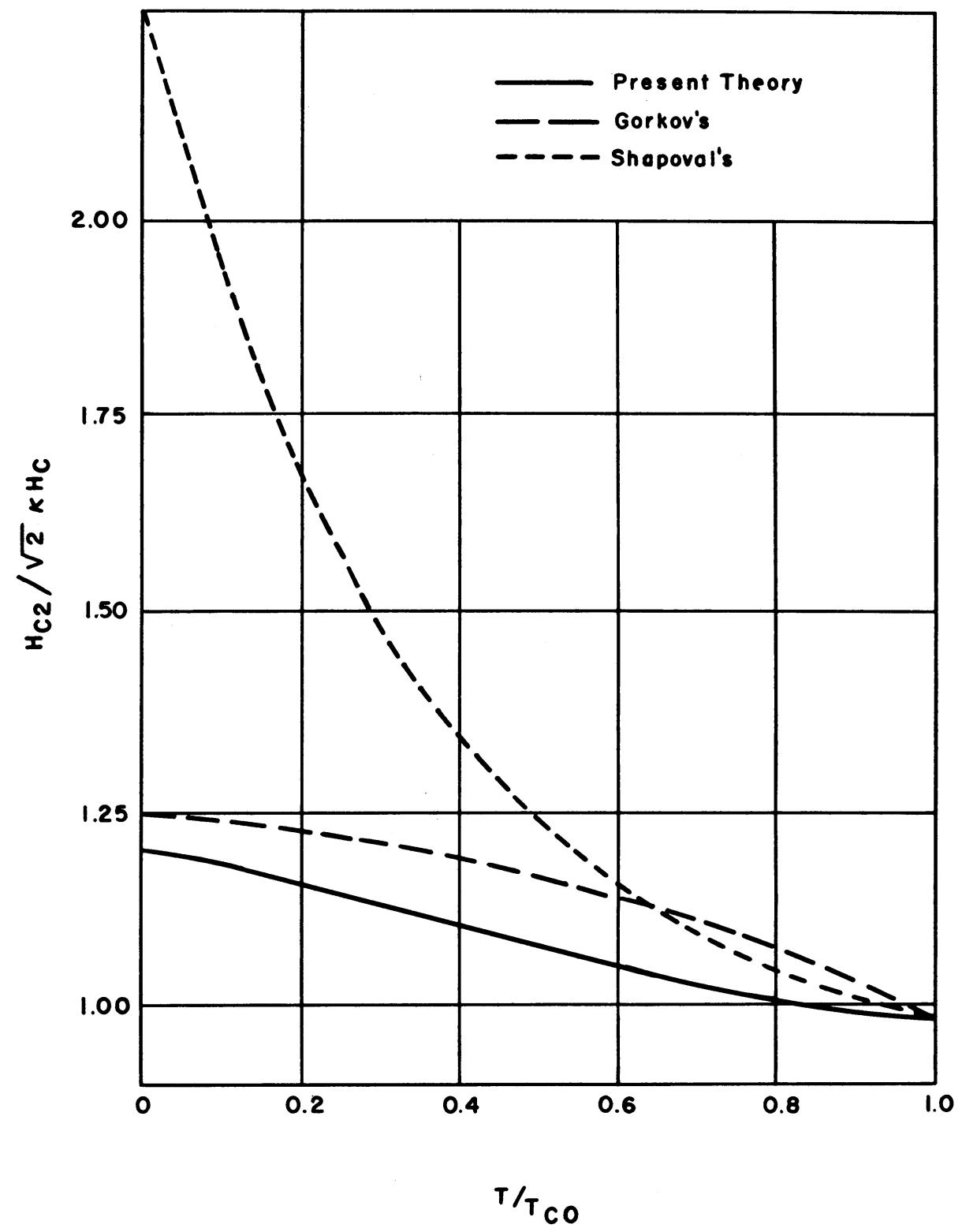

FIGURE 1

The ratio $H_{c 2} / \sqrt{2} \kappa H_{c}$ is depicted as a function of the temperature, and compared with Gorkov's and Shapovals theories.

sists to the upper critical field $H_{\mathrm{c} 2}$ where the superconductive correlation disappears at a second order phase transition. Following Abrikosov, we seek the solution of equations (5) and (6) by the use of the following variational function

$$
\Delta^{+}(r)=\sum_{n=-\infty}^{\infty} C_{n} e^{i k n y} \psi_{n}(x)
$$

where

$$
\psi_{n}(x)=\exp \left\{-e H_{0}\left(x-\frac{k n}{2 e H_{0}}\right)^{2}\right\}
$$

and $H_{0}$ is the external field. 
Substituting this expression in equation (6) we obtain

where

$$
\vec{j}(r)=-\frac{e \tau_{t r} N}{4 m \pi T} \mathcal{g}(\rho)\left\{4 e \vec{A}|\Delta|^{2}-\frac{1}{i}\left(\Delta^{+} \vec{\partial} \Delta-\Delta \vec{\partial} \Delta^{+}\right)\right\}
$$

$$
g(z)=\sum_{n=0}^{\infty} \frac{1}{\left(n+\frac{1}{2}+z\right)^{2}} \quad \text { and } \quad \rho=\frac{\tau_{t r} v^{2}}{6 \pi T} e H_{0}
$$

Solving the equation rotrot $\vec{A}=4 \pi \vec{j}$, we have

$$
\frac{\partial A_{y}}{\partial x}=H=H_{0}-\frac{e \tau_{t r} N}{m T} g(\rho)|\Delta|^{2} .
$$

We can further carry out similar calculations to those given by Abrikosov and find

$$
\frac{\tau_{t r} e v^{2}}{6 \pi T} g(\rho)\left(H_{c 2}-H_{0}\right) \overline{|\Delta|^{2}}+\frac{1}{8(\pi T)^{2}}\left\{\frac{4 \pi N}{3 m}\left(e \tau_{t r} v \mathcal{B}(\rho)\right)^{2}-f_{1}(\rho)\right\} \overline{|\Delta|^{4}}=0,
$$

where

$$
f_{1}(z)=\sum_{n=0}^{\infty} \frac{1}{\left(n+\frac{1}{2}+z\right)^{3}}-z \sum_{n=0}^{\infty} \frac{1}{\left(n+\frac{1}{2}+z\right)^{4}}
$$

The magnetic induction and the free energy are given by

$$
B=\bar{H}=H_{0}-\frac{\left(H_{c 2}-H_{0}\right)}{\left(2 \kappa_{2}^{2}(T)-1\right) \beta},
$$

and

$$
\Delta F_{S H}=\frac{1}{4 \pi}\left[B^{2}-\frac{\left(H_{c 2}-B\right)^{2}}{\left(2 \kappa_{2}^{2}(T)-1\right) \beta+1}\right] \text {, }
$$

where

$$
\beta=\overline{|\Delta|^{4}} /\left(\overline{|\Delta|^{2}}\right)^{2}\left(=1.18^{1}\right)
$$

and

$$
\kappa_{2}(T)=\left(\frac{3 m f_{1}(\rho)}{8 \pi N}\right)^{1 / 2}\left(e \tau_{t r} v g(\rho)\right)^{-1}
$$

Asymptotic equations for $\kappa_{2}$ are:

$$
\begin{aligned}
\kappa_{2} & =0.69 \kappa\left(1+\frac{10}{3}\left(\frac{\pi T}{\Delta_{00}}\right)^{2}\right) \\
& =0.69 \kappa\left(1+\frac{31}{3} \frac{T^{2}}{T_{\mathrm{c} 0}^{2}}\right), \quad \text { for } \quad T \ll T_{\mathrm{c} 0}, \\
& =\kappa(1-0.087 \theta), \quad \text { for } \quad T_{\mathrm{c} 0}-T \ll T_{\mathrm{c} 0} .
\end{aligned}
$$


It is interesting to note that Abrikosov's structure at arbitrary temperatures is described by the use of two parameters $\kappa_{1}(T)$ and $\kappa_{2}(T)$ which appear in the expressions for the upper critical field $H_{c 2}$ and for the magnetization $M$ respectively as

$$
H_{c 2}=\sqrt{2} \kappa_{1}(T) H_{c}
$$

and

$$
-4 \pi M / H_{c 2}-H_{0}=1 / 1.18\left(2 \kappa_{2}^{2}(T)-1\right) \text {. }
$$

The temperature dependence of $\kappa_{2}$ has been calculated numerically and is shown in Figure 2 with $\kappa_{1}$.

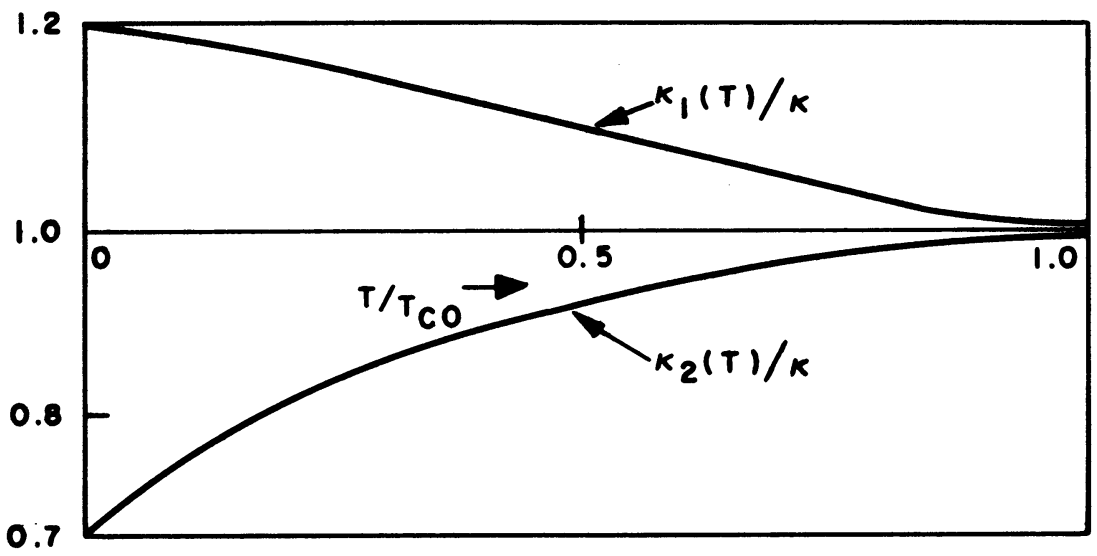

FIGURE 2

The temperature dependence of the two parameters $\kappa_{1}(T)$ and $\kappa_{2}(T)$ are given.

We find a relation $\kappa_{1}(T) \geqq \kappa \geqq \kappa_{2}(T)$ where the equality holds only at $T=T_{\mathrm{c} 0}$. From the general theory of the second order phase transition [11] we know that the order of the transition changes as $\kappa_{2}(T)$ passes the critical value $1 / \sqrt{2}$. We expect a peculiar thermodynamical behavior for superconductors with $\kappa_{1}$ and $\kappa_{2}$ satisfying $\kappa_{1}>1 / \sqrt{2}>\kappa_{2}$. For these superconductors (which may be called type III superconductors) the mixed state terminates in a first order phase transition and the upper critical field calculated in $\S 3$ is interpreted as a supercooling critical field. The magnetization curve is rather schematically presented in Figure 3. A superconductor having $\kappa$ slightly larger than $1 / \sqrt{2}$ belongs to the second type at the critical temperature. As the temperature decreases the jump in the specific heat at the transition increases and becomes unbounded as $\left(T-T_{1}\right)^{1 / 2}$ at the critical point $\left(H_{1}, T_{1}\right)$ in the $\left(H_{c 2}-T\right.$ diagram $)$ which is determined from the equation

$$
\left(\frac{3 m f_{1}(\rho)}{4 \pi N}\right)^{1 / 2}\left(e \tau_{t r} v g(\rho)^{-1}=1 .\right.
$$

At lower temperatures $\left(T<T_{1}\right)$ the superconductor behaves as one of the third type.

\section{Concluding Remarks}

We have seen above that Abrikosov's theory is valid in a rather wide temperature range if one uses two parameters $\kappa_{1}(T)$ and $\kappa_{2}(T)$. The temperature dependence of these parameters was numerically calculated. A peculiar deviation from his theory is predicted for superconductors with $\kappa_{1}$ and $\kappa_{2}$ satisfying $\kappa_{1}>1 / \sqrt{2}>$ $\kappa_{2}$. The superconducting alloys studied by Kinsel et al [10] belong to this type. Therefore, more accurate observation of their thermodynamical behavior is desirable. We suggest here that superconductors having a 

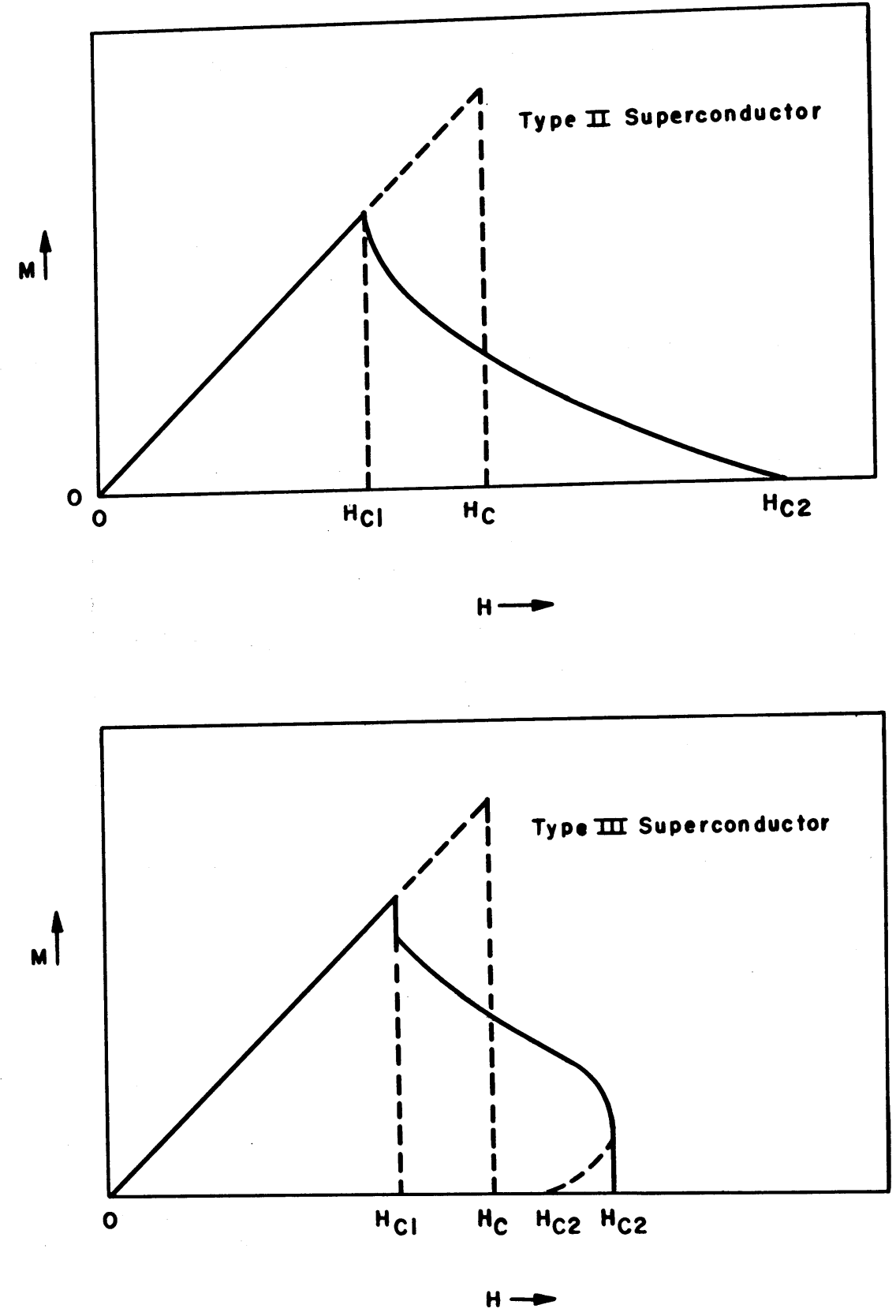

FIGURE 3

Magnetization curves for superconductors of the second type and of the third type are schematically presented. For the superconductors of the third type $\boldsymbol{H}_{\mathrm{c} 2}$ given in $\$ 3$ corresponds to a supercooling critical field.

large Pauli paramagnetism may behave similarly. In the second part of this work Abrikosov's structure in the vicinity of $H_{\mathrm{c} 1}$ as well as the relation between the ordering parameter $\Delta$ and the gap in the energy spectrum will be discussed.

In conclusion the author wishes to thank Dr. T. Tsuneto for informative conversations on type II superconductors and interesting discussions.

\section{Appendix}

In the presence of a magnetic field the Fourier transform of the Green's function is formally given as

$$
G_{\omega}(\rho)=(i \hat{\omega}-e \vec{v} \vec{A}+\xi)^{-1}
$$


where $\vec{A}$ is the vector potential and $v$ the velocity of the electron.

The correction to the vertex is obtained by summing up ladder type diagrams, or equivalently, by solving the following integral equation [2]

$$
K\left(p_{1}, p_{2}\right)=G_{\omega}\left(p_{1}\right) G_{-\omega}\left(p_{2}\right)\left(1+\frac{n}{(2 \pi)^{3}} \int|u|^{2} K\left(p_{1}^{\prime}, p_{2}^{\prime}\right) d^{3} p^{\prime}\right) .
$$

We assume here the scattering due to impurity atoms is isotropic for simplicity. Putting $\kappa=G_{\omega} G_{-\omega} \eta_{\omega q}$ where $q=p_{1}+p_{2}$ we obtain:

$$
\eta_{\omega_{q}}=1+\eta_{\omega_{q}} I
$$

where

$$
\begin{aligned}
I= & \frac{1}{2 \pi} \int d \xi d \Omega\left\{\frac{1}{(\widetilde{\omega} i-e \vec{v} \vec{A}+\xi)(\widetilde{\omega} i-e \vec{v} \vec{A}-\xi+v q)}\right\} \\
= & \frac{1}{2 \pi \tau} \int d \xi d \omega\left(\frac{1}{\widetilde{\omega} i+\xi}+\frac{1}{\widetilde{\omega} i+\xi} e \vec{v} \vec{A}_{p} \frac{1}{\widetilde{\omega} i+\xi+p v}\right. \\
& \left.+\frac{1}{\widetilde{\omega} i+\xi} e \vec{v} \vec{A}_{p} \frac{1}{\widetilde{\omega} i+\xi+p v} e \vec{v} \vec{A}_{p^{\prime}-p} \frac{1}{\widetilde{\omega} i+\xi+p^{\prime} v}\right) \\
& +\left(\frac{1}{\widetilde{\omega} i-\xi+v q}+\frac{1}{\widetilde{\omega} i-\xi+v q} e \vec{v} \vec{A}_{p} \frac{1}{\widetilde{\omega} i-\xi+v q-p v}\right. \\
& \left.+\frac{1}{\widetilde{\omega} i-\xi+v q} e \vec{v} \vec{A}_{p} \frac{1}{\widetilde{\omega} i-\xi+v q-v p} e \vec{v} \vec{A}_{p^{\prime}-p} \frac{1}{\widetilde{\omega} i-\xi+v q-v p}\right) \\
= & \frac{1}{2 \widetilde{\omega} \tau}\left\{1-\frac{1}{3} \tau^{2} v^{2}(\vec{q}-2 e \vec{A})^{2}+\frac{1}{5} \tau^{4} v^{4}(\vec{q}-2 e \vec{A})^{4}+\frac{1}{15} \tau^{4} e^{2} v^{2} H^{2}\right\}
\end{aligned}
$$

and

$$
H=\nabla \times A .
$$

In equation $(A-5)$ we can show that the third and the fourth terms are of higher order in $\left(1 / \xi_{0}\right)$ and can be neglected. We finally obtain

$$
\eta_{\omega_{q}}=\left\{1-\frac{1}{2 \tau \widetilde{\widetilde{\omega}}}\left(1-\frac{\tau^{2}}{3} v^{2}(q-2 e A)^{2}\right)\right\}^{-1}+0\left(1 / \xi_{0}\right) .
$$

Using the above expression for $\eta_{q} \omega$ one can easily see that the magnetic field is introduced into the theory by replacing $q$ by $q \pm 2 e A$ as stated in $\S 2$. For pure superconductors we can show that such a simple procedure is not valid.

\section{References}

1. A. A. ABRIKOSOV, JETP 32, 1442 (1957); Soviet Phys. JETP 5, 1174 (1957).

2. L. P. GOR'KOV, JETP 36, 1918 (1959); 37, 1407 (1959); Soviet Phys.-JETP 9, 1364 (1959); 10, 998 (1960).

3. Y. NAMBU and S. F. TUAN, Phys. Rev. 128, 2622 (1962). 
4. L. TEWORDT, Phys. Rev. 132, 595 (1963).

5. N. R. WERTHAMER, Phys. Rev. 132, 663 (1963).

6. L. P. GOR'KOV, JETP 34, 735 (1958); Soviet Phys.-JETP 7, 505 (1958).

7. L. P. GOR'KOV, JETP 37, 835 (1959); Soviet Phys.-JETP 10, 593 (1960).

8. E. A. SHAPOVAL, JETP 41, 877 (1961); Soviet Phys.-JETP 14, 628 (1962).

9. N. R. WERTHAMER, Rev. Mod. Phys. 36, 116 (1964).

10. T. KINSEL, E. A. LYNTON and B. SERIN, Rev. Mod. Phys. 36, 105 (1964).

11. N. R. WERTHAMER, Phys. Rev. 132, 2440 (1963).

12. L. D. LANDAU and E. M. LIFShitZ, Statistical Physics, Pergamon Press, New York (1958). 